ARQGA / 955

\title{
CARCINOMA ESPINOCELULAR DE CANAL ANAL: análise de 11 casos
}

\author{
Ana Paula GUIMARÃES*, Délcio MATOS**, \\ Roberto SEGRETO*** e Nora Manoukian FORONES****
}

RESUMO - Racional - O câncer de canal anal é um tipo raro de neoplasia, sendo responsável por 4\% dos tumores de intestino grosso. São descritos os aspectos clínicos e o tratamento dos pacientes com câncer escamoso desta região. Casuística - Analisaram-se, retrospectivamente, 11 pacientes com carcinoma espinocelular de canal anal. Nove pacientes eram do sexo feminino e dois do masculino. A idade média dos pacientes foi de 57,6 anos (variação 35-82 anos). Resultados - Os sintomas mais freqüentes foram sangramento retal, tumoração e dor local. Seis pacientes tinham lesão benigna prévia local e dois tinham doença metastática na ocasião do diagnóstico. Todos foram submetidos a quimioterapia sistêmica com 5-fluorouracil e mitomicina. Após o tratamento quimio e radioterápico, quatro apresentavam doença residual e foram submetidos a ressecção abdômino-perineal. Três pacientes apresentaram recidiva e quatro morreram pela evolução da própria doença. Conclusão - A maioria dos doentes era do sexo feminino. A químio e a radioterapia podem ser tratamentos curativos nos pacientes com doença localizada; no entanto, aqueles com doença residual devem ser submetidos a ressecção abdômino-perineal. Embora o câncer espinocelular de canal anal seja doença curável, quatro pacientes foram a óbito por terem sido diagnosticados em fase avançada.

DESCRITORES - Carcinoma de células escamosas. Neoplasias do ânus.

\section{INTRODUÇÃO}

O câncer de canal anal apresenta-se como um tipo infreqüente de neoplasia. Nos EUA observa-se que cerca de $1 \%$ a $2 \%$ dos cânceres de intestino grosso e 3,9\% dos tumores anorretais são de canal anal ${ }^{(16,33)}$. O tipo histológico mais comum é o espinocelular, observado em até $47 \%$ dos casos, seguido pelos tipos, cloacogênico, adenocarcinoma e, mais raramente, melanoma ou adenocarcinoma mucinoso ${ }^{(33)}$.

Dados epidemiológicos evidenciam diferentes fatores de risco para o desenvolvimento da patologia como, as doenças sexualmente transmissíveis, o coito anal, o homossexualismo, o tabagismo, as doenças perianais e a imunodepressão ${ }^{(7,10,14,18,19)}$.

$\mathrm{O}$ canal anal se estende do reto até a pele perianal. O estadiamento deste câncer obedece ao sistema UICC
(International Union Against Cancer) de 1987, onde T equivale ao tamanho e à invasão do tumor nas diferentes camadas do órgão, $\mathrm{N}$ faz relação a invasão de linfonodos e $\mathrm{M}$ a presença ou não de metástases (Quadro 1). Os tumores menores que 2 $\mathrm{cm}$ e os bem diferenciados têm melhor prognóstico ${ }^{(25,33)}$.

No que se refere ao tratamento, a cirurgia de ressecção abdômino-perineal de reto era o tratamento de escolha ${ }^{(27)}$; hoje se opta em primeira linha pela radioterapia, associada ou não à quimioterapia ${ }^{(3,4,6,8,12,22)}$.

Neste estudo foram avaliados os pacientes com carcinoma espinocelulares de canal anal, tratados e acompanhados no ambulatório do Hospital São Paulo da Universidade Federal de São Paulo - Escola Paulista de Medicina UNIFESP-EPM.

\footnotetext{
* Aluna do Curso de Especialização em Gastroenterologia Clínica da Universidade Federal de São Paulo - UNIFESP-EPM, São Paulo, SP.

** Professor Livre-Docente da Disciplina de Gastroenterologia Cirúrgica da UNIFESP-EPM.

*** Professor Livre-Docente do Setor de Radioterapia da UNIFESP-EPM.

**** Professor Adjunto da Disciplina de Gastroenterologia Clínica da UNIFESP-EPM.

Endereço para correspondência: Dra. Nora Manoukian Forones - Rua Botucatu 740, $2^{\circ}$ andar - 04023-900 - São Paulo, SP. e-mail: nora@gastro.epm.br
} 
QUADRO 1 - Estádio clínico do carcinoma espinocelular de canal anal (segundo UICC, 1987)

\begin{tabular}{|c|c|c|c|}
\hline \multicolumn{4}{|c|}{ Critérios para estadiamento TNM } \\
\hline T1 - Tum & \multicolumn{3}{|c|}{ Tumor $<2 \mathrm{~cm}$} \\
\hline T2 - Tum & \multicolumn{3}{|c|}{ Tumor $>2 \mathrm{e}<5 \mathrm{~cm}$} \\
\hline T3 - Tum & \multicolumn{3}{|c|}{ Tumor $>5 \mathrm{~cm}$} \\
\hline T4 - Tum & \multicolumn{3}{|c|}{ Tumor invadindo órgãos adjacentes } \\
\hline N0 - $\quad$ Ausê & \multicolumn{3}{|c|}{ Ausência de linfonodos envolvidos } \\
\hline N1 - Linfo & \multicolumn{3}{|c|}{ Linfonodos perirretais envolvidos } \\
\hline $\begin{array}{ll}\mathrm{N} 2- & \text { Linfo } \\
\text { envo }\end{array}$ & \multicolumn{3}{|c|}{$\begin{array}{l}\text { Linfonodos ilíacos internos unilaterais ou inguinais } \\
\text { envolvidos }\end{array}$} \\
\hline N3 - $\quad \begin{array}{l}\text { Linf } \\
\text { bilat }\end{array}$ & \multicolumn{3}{|c|}{$\begin{array}{l}\text { Linfonodos perirretais + inguinais e/ou ilíacos internos } \\
\text { bilaterais e/ou inguinais envolvidos }\end{array}$} \\
\hline M0 - ausêr & \multicolumn{3}{|c|}{ ausência de metástases a distância } \\
\hline M1 - prese & \multicolumn{3}{|c|}{ presença de metástase a distância } \\
\hline Estádio I & $\mathrm{T} 1$ & No & M0 \\
\hline Estádio II & $\mathrm{T} 2-3$ & No & M0 \\
\hline \multirow[t]{2}{*}{ Estádio IIIA } & $\mathrm{T} 4$ & No & M0 \\
\hline & $\mathrm{T} 1-3$ & $\mathrm{~N} 1$ & M0 \\
\hline \multirow[t]{2}{*}{ Estádio IIIB } & $\mathrm{T} 4$ & $\mathrm{~N} 1$ & M0 \\
\hline & $\mathrm{T}$ qualquer & $\mathrm{N} 2-3$ & M0 \\
\hline Estádio IV & $\mathrm{T}$ qualquer & N qualquer & M1 \\
\hline
\end{tabular}

\section{CASUÍSTICA E MÉTODOS}

Realizou-se análise retrospectiva de 11 casos, sendo 9 mulheres e 2 homens, atendidos no setor de Oncologia da Disciplina de Gastroenterologia da UNIFESP-EPM, no período de janeiro de 1995 a junho de 1999. A idade média dos pacientes foi de 56,8 anos (35 a 82 anos). Todos apresentavam exame anatomopatológico com diagnóstico de carcinoma espinocelular (CEC). Analisaram-se as histórias clínicas, o tratamento realizado (radioterapia, quimioterapia e/ou cirurgia), o aparecimento de recurrência e a sobrevida (Quadro 2). Entre os antecedentes pessoais, avaliaram-se a promiscuidade sexual, a doença anterior em região anal, o tabagismo e o uso de drogas endovenosas.

QUADRO 2 - Aspectos clínicos e terapêuticos dos doentes com carcinoma espinocelular de canal anal

\begin{tabular}{|lll|}
\hline Sexo & $\mathrm{F}$ & $\mathrm{N}=9$ \\
& $\mathrm{M}$ & $\mathrm{N}=2$ \\
\hline Idade (anos) & média & 56,8 \\
\hline Tratamento & Qt + Radio & $\mathrm{N}=10$ \\
\hline Cirurgia & & $\mathrm{N}=4$ \\
\hline Recurrência & & $\mathrm{N}=3$ \\
\hline Óbito & $\mathrm{N}=4$ \\
\hline
\end{tabular}

\section{RESULTADOS}

Foi observada maior prevalência do carcinoma espinocelular de canal anal no sexo feminino em relação ao sexo masculino (4,5:1).

$\mathrm{Na}$ investigação da história clínica, observou-se presença de sangramento em $91 \%$, mudança das características das fezes (afilamento) em 54\%, sensação de corpo estranho em região anal em $36 \%$, dor em região anal em $36 \%$, prurido anal em $27 \%$, incontinência fecal em $18 \%$ e emagrecimento em $45 \%$.

Quanto aos antecedentes pessoais, três pacientes eram tabagistas, um fazia uso de drogas endovenosas e apenas um paciente relatou promiscuidade sexual. Dos 11 pacientes 6 possuíam lesão prévia em região anal, sendo 4 com lesões sugestivas de HPV e 2 com botões hemorroidários.

Quanto ao estádio, houve grande variação: três pacientes eram estádio II, cinco eram estádio III e dois estádio IV (Quadro 3); um dos pacientes abandonou o tratamento antes mesmo de terminar o estadiamento. Todos os pacientes foram submetidos a quimioterapia com 5-fluorouracil (5-FU) e mitocina, nas dosagens de $15 \mathrm{mg} / \mathrm{m}^{2} \mathrm{de}$ mitomicina no primeiro dia e de $1 \mathrm{~g} / \mathrm{m}^{2}$ de 5 -FU por dia, por infusão contínua por 4 dias $\left(1^{\circ}\right.$ ao $4^{\circ}$ dia). A radioterapia realizada foi externa, nas dosagens de 4500-5500cGy divididas em 25 sessões, de início, no $1^{\circ}$ dia de quimioterapia. A toxicidade observada foi leve a moderada, sendo náuseas e ardor no local da punção, as principais queixas. Não foram observadas leucopenias severas (glóbulos brancos inferiores a $2000 / \mathrm{mm}^{3}$ ) ou plaquetopenia inferior a 50000 / $\mathrm{mm}^{3}$. Também não foram observados diarréias ou vômitos que justificassem internação do paciente para reposição hidroeletrolítica (toxicidade 3 ou 4).

Quatro pacientes foram encaminhados à cirurgia para ressecção de tumor residual. A doença foi considerada irressecável em um deles,

QUADRO 3 - Estádio clínico dos doentes com carcinoma espinocelular de canal anal (segundo UICC, 1987)

\begin{tabular}{|llc|}
\hline Pacientes & TNM & Estádio \\
\hline IC & T1N0M0 & II \\
\hline DBT & T1N0M0 & II \\
\hline DSS & T1N0M0 & II \\
\hline LCS & T1N0M0 & IIIA \\
\hline ACO & T1N0M0 & IIIA \\
\hline ECO & T4N0M0 & IIIA \\
\hline CS & T1N1M0 & IIIA \\
\hline EASM & T1N1M1 & IIIB \\
\hline RDT & T4N1M1 & IV \\
\hline MB & T4N1M1 & IV \\
\hline
\end{tabular}


sendo submetido a novas sessões de quimioterapia com 5-FU e mitocina. Um dos doentes que desenvolveu recurrência, também foi submetido a quimioterapia com 5 -FU e cisplatina. Esta última foi prescrita na dosagem de $75 \mathrm{mg} / \mathrm{m}^{2}$ no $1^{\circ}$ dia de quimioterapia, associada ao 5-FU nas mesmas dosagens que no esquema anterior. Neste esquema não foi observada toxicidade renal, mas náuseas e vômitos foram sintomas freqüentes, que não impediram a continuidade do tratamento. O uso de antieméticos como o ondansetron associado a dexametasona torna-se necessário na realização deste esquema.

Dois doentes abandonaram o tratamento, sendo que um deles não tinha terminado o estadiamento ou iniciado qualquer terapêutica. Entre os três que desenvolveram recurrência, um foi a óbito após 3 meses, o outro após 2 anos e o terceiro continua vivo com 40 meses de acompanhamento, após o diagnóstico da doença. Entre os doentes com doença metastática, a sobrevida foi de 18 meses, em média. Dois destes tinham doença metastática na ocasião do diagnóstico e também foram a óbito. Os demais doentes estão em acompanhamento no Serviço por um período que varia entre 20 e 60 meses.

\section{DISCUSSÃO}

O carcinoma espinocelular de canal anal é muito menos freqüente que o adenocarcinoma de reto; por isso, durante 5 anos observaram-se apenas 11 pacientes com tumores nesta região. GALLÉN e PLA ${ }^{(14)}$ também observaram apenas 20 casos, em 14 anos, no Hospital Delmar, em Barcelona.

A população acometida por esta neoplasia foi predominantemente do sexo feminino (82\%), semelhante à descrita por RICH et al. ${ }^{(31)}$, OLIVEIRA $^{(27)}$, HABR-GAMA et al. ${ }^{(15)}$, que observaram maior freqüência deste câncer na população do sexo feminino. GABRIEL ${ }^{(13)}$, em 1941, e MORSON ${ }^{(26)}$, em 1959, demonstraram que, ao contrário do adenocarcinoma de reto, o carcinoma epidermóide de canal anal tinha distribuição semelhante nos dois sexos. GABRIEL ${ }^{(13)}$ observou, também, que os tumores no sexo masculino eram menos agressivos que nas mulheres.

Em relação à idade, observou-se grande variação, sendo $36 \%$ com idade inferior a 50 anos, ao contrário do observado no adenocarcinoma de reto, onde a maior parte da população tem mais de $50 \operatorname{anos}^{(33)}$.

Muitos autores relacionam esta neoplasia a doenças prévias locais que causariam irritação crônica no canal anal, como condilomas, fístulas, hemorróidas ou abscessos ${ }^{(1,7)}$. Entre os doentes desta série, observaram-se que $27 \%$ apresentavam doença local por papiloma vírus e $18 \%$ por hemorróidas. Apenas um paciente fazia uso de drogas endovenosas. Quanto à promiscuidade, torna-se difícil a verdadeira investigação devido a falta de relatos dos pacientes que omitem dados referentes a suas atividades sexuais.

O diagnóstico de carcinoma epidermóide é relativamente fácil ${ }^{(3,}$ ${ }^{11)}$, porém a semelhança dos sintomas com os quadros hemorroidários (dor local, sangramento e tumoração anal), a negligência médica em não examinar a região anal ou ainda o desconhecimento da patologia, fazem com que o diagnóstico seja feito, em muitos casos, em fases avançadas da doença.

A disseminação do CEC de canal anal é diferente da descrita no adenocarcinoma de reto. Neste, a disseminação é principalmente hematogênica ou por contiguidade, com formação de metástase pulmonar e hepática. Ao contrário, no CEC as metástases nestes órgãos são raras e a disseminação linfática para gânglios inguinais e mesmo mesentéricos, são mais freqüentes. Esta via de disseminação torna a radioterapia local e da região inguinal necessária ao tratamento, independente da ressecção do tumor. Devido a este fato, ao tipo de cirurgia mutiladora necessária para ressecção destes tumores com conseqüente colostomia definitiva e aos bons resultados observados em outras neoplasias espinocelulares, como nos tumores de laringe, a quimioterapia e a radioterapia tornaramse o tratamento de eleição destes doentes. Após o término do tratamento, a lesão local deve ser avaliada; se persistente deverá, então, ser ressecada cirurgicamente ${ }^{(21,23,30)}$. A principal droga quimioterápica usada tem sido a 5 -FU combinada com a mitocina e/ou cisplatina por 4 a $5 \operatorname{dias}^{(9,24,28,29,30,32,33)}$. Associada à quimioterapia, realiza-se a radioterapia com dose de radiação entre 4500 e 5500 cGy, dividida em 5 semanas ${ }^{(20,30,31,32)}$. Nos estádios iniciais, menores doses de radiação (3000cGy) têm bons resultados com menos efeitos colaterais ${ }^{(34,35)}$.

Com esta terapêutica, observa-se índice de sobrevida de 70\%, após 5 anos. A doença confinada ao plano muscular está associada a menor risco de recidiva local e menor mortalidade ${ }^{(17)}$. A mitomicina parece ser uma das drogas de melhor eficácia e menor toxicidade. Alguns autores ${ }^{(28,36)}$ preconizam o uso de outras drogas, como a cisplatina ao invés da mitocina nos doentes que não tiveram resposta total ou mesmo como primeira droga associada ao 5-FU, nos doentes com tumores avançados. No entanto, a sobrevida não parece ser maior nos doentes que usaram cisplatina e os efeitos colaterais são superiores. Por isso preferiu-se o esquema 5-FU e mitocina como primeira escolha, sendo a cisplatina indicada nos casos de recidiva não-ressecável do tumor. Nos casos de doença residual optou-se por ressecção tumoral. Mesmo nos casos avançados, a cirurgia após radio e quimioterapia pode curar $50 \%$ dos doentes.

Nos idosos, sem condições cirúrgicas ou nos doentes com tumores in situ, o tratamento local com laser pode ser indicado ${ }^{(2,37)}$.

Conclui-se que o carcinoma espinocelular de canal anal teve maior incidência no sexo feminino. A quimioterapia associada à radioterapia pode ser tratamento curativo nos doentes com doença localizada; no entanto, pacientes com doença residual devem ser submetidos a ressecção abdômino-perineal. Embora o câncer espinocelular de canal anal seja doença curável, quatro pacientes foram a óbito por terem sido diagnosticados em fase avançada. 
Guimarães AP, Matos D, Segreto R, Forones NM. Squamous cell carcinoma of the canal anal: analysis of 11 patients. Arq Gastroenterol 2001;38(1):9-12.

ABSTRACT - Background - Anal cancer is an uncommon malignancy accounting for only a small (4\%) percentage of intestinal cancer. The authors described the clinical aspects and the treatment of the patients with squamous cell carcinoma of the canal anal. Patients - Eleven patients with squamous cell carcinoma treated among 1995 and 1999, were analyzed retrospectively. Nine were women and two were men. The mean age was 57.6 years old (range 35-82 years old). Results - The most common symptoms were rectal bleeding, local tumor and pain. Six of them had previous anal benign disease and two had metastases at the diagnosis. All were submitted to systemic chemotherapy with 5-fluorouracil and mitomicin and radiotherapy with 4500cGy. Four patients had residual disease after chemo radiation and salvage surgery with abdominoperineal resection was done. Three patients had recurrence and four died from the disease. Conclusion - Most of our patients were women. The chemo radiation can be a curable treatment in patients with local disease; conversely in patients with residual disease, abdominoperineal resection must be done. Although anal cancer is an often curable disease, four patients died because the diagnosis was done in advanced stage.

HEADINGS - Carcinoma, squamous cell. Anus neoplasms.

\section{REFERÊNCIAS BIBLIOGRÁFICAS}

1. Ablin RJ, Stein-Werblowsky R. Sexual behaviour and increased anal cancer. Immunol Cell Biol 1997;75:181-3.

2. Bandieramonte G, Bono A, Zurrida S, Bartoli C, de Palo G. Laser surgery for small perianal neoplasms. Eur J Cancer 1993;29A:1528-31.

3. Boman BM, Moertel CG, O'Connell MJ, Scott M, Weiland LH, Beart RW, Gunderson LL, Spencer RJ. Carcinoma of the anal canal: a clinical and pathologic study of 188 cases. Cancer 1984;54:114-25.

4. Cantril ST, Green JP, Schall GL, Schaupp WC. Primary radiation therapy in the treatment of anal carcinoma. Int J Radiat Oncol Biol Phys 1983;9:1271-8.

5. Cummings B, Keane T, Thomas G. Results and toxicity of the treatment of anal canal carcinoma by radiation therapy or radiation therapy and chemotherapy. Cancer 1984;54:2062-8.

6. Cummings BJ. The role of radiation therapy with 5-fluorouracil in anal canal cancer. Sem Rad Oncol 1997;7:306-12.

7. Daling JR, Weiss NS, Hislop TG, Maalen C, Coates RJ, Sherman KJ, Ashley RL, Beague M, Ryan JA, Corey L. Sexual practices, sexually transmitted diseases, and the incidence of anal cancer. N Engl J Med 1987;317:973-7.

8. Enker WE, Heilwell M, Janov AJ, Quan SH, Magill G, Steams MW Jr., Shank B, Leaming R, Sternberg SS. Improved survival in epidermoid carcinoma of the anus in association with preoperative multidisciplinary therapy. Arch Surg 1986;121:1386-90.

9. Flam M, John M, Pajak TF, Petrelli N, Myerson R, Doggett S, Quirey J, Rotman M, Kerman H, Coia L, Murray K. Role of mitomycin in combination with fluorouracil and radiotherapy, and of salvage chemoradiation in the definitive nonsurgical treatment of epidermoid carcinoma of the anal canal: results of a phase III randomized intergroup study. J Clin Oncol 1996;14:2527-39.

10. Frisch M, Fenger C, VanDen Brule A, Sorensen P, Meijer C, Walboomers J, Adam $\mathrm{H}$, Melbye M, Glimelius B. Variants of squamous cell carcinoma of the anal canal and perianal skin and their relation to human papilomaviruses. Cancer Res 1999;59:753-7.

11. Fuchshuber PR, Rodriguez-Bigas M, Weber T, Petrelli NJ. Anal canal and perianal epidermoid cancers. J Am Coll Surg 1997;185:494-505.

12. Fung CY, Willett CG, Efird JT. Chemoradiotherapy for anal carcinoma: what is the optimal radiation dose? Radiat Oncol Investig 1994;2:152-6.

13. Gabriel WB. Squamous-cell carcinoma of the anus and anal canal. Proc R Soc Med 1941;34:139-46.

14. Gallen M, Pla J, Miguel A, Ibeas R, Charles J, Fabregat X. Anal carcinoma: a 14 year experience. Rev Esp Enferm Dig 1997;89:23-8.
15. Habr-Gama A, Sousa AHS, Nadalin W, Gansl R, Silva JH, Pinotti HW. Epidermoid carcinoma of the canal anal. Dis Colon Rectum 1989;32:773-7.

16. Harter KW, Ahlgren J. Cancer of the canal anal and anal margin. In: Ahlgren J, Macdonald J, editors. Gastrointestinal oncology. Philadelphia: Lippincott; 1992. p.437-48.

17. Hill J, Meadows H, Meadows C, Houghton J, Northover J. UKCCCR anal cancer trial salvage surgery study. Colorectal Dis 2000;2:5.

18. Hoffman R, Krieg R, Klencke B. Outcome and treatment tolerance for HIV-positive patients with anal cancer based on pretreatment CD4 count. Int J Radiat Oncol Biol Phys 1998;42:A-80, 164.

19. Holland JM, Swift PS. Tolerance of patients with human immunodeficiency virus and anal carcinoma to treatment with combined chemotherapy and radiation therapy. Radiology 1994;193:251-4.

20. James RD, Pointon RS, Martin S. Local radiotherapy in the management of squamous carcinoma of the anus. Br J Surg 1985;72:282-5.

21. John M, Pajak T, Flam M, Hoffman J, Markae E, Wolkov H, Paris K. Dose escalation in chemoradiation for anal cancer: preliminary results of RTOG 92-08. Cancer J Sci Am 1996;2:205-11.

22. Leichman L, Nigro N, Vaitkevicius VK, Cousidine B, Burokec T, Bradley G, Seidel HG, Olchowski S, Cummings G, Leidman C. Cancer of the anal canal: model for preoperative adjuvant combined modality therapy. Am J Med $1985 ; 78: 211-5$.

23. Longo WE, Vernava AM, Wade TP, Coplin MA, Virgo KS, Johnson FE. Recurren squamous cell carcinoma of the anal canal: predictors of initial treatment failure and results of salvage therapy. Ann Surg 1994;220:40-9.

24. Martenson JA, Lipsitz SR, Lefkopoulou M, Engstrom PF, Dayal YY, Coban CD, Oken MM, Haller DJ. Results of combined modality therapy for patients with anal cancer (E7283): an Eastern Cooperative Oncology Group study. Cancer 1995;76:1731-6.

25. Mitchell E. Carcinoma of the anal region. Sem Oncol 1988;15:146-53.

26. Morson BC. The pathology and results of treatment of cancer of the anal region. Proc R Soc Med 1959;53:410-5.

27. Oliveira D. Anal cancer in northeast Brazil. Dis Colon Rectum 1976;19:18-9.

28. Papillon J, Mayer M, Montbarbon JF, Gerard JP, Chassard JL, Bailly C. A new approach to the management of epidermoid carcinoma of the anal canal. Cancer $1983 ; 51: 1830-7$

29. Peddada AV, Smith DE, Rao AR, Frost DB, Kayan AR. Chemotherapy and lowdose radiotherapy in the treatment of HIV-infected patients with carcinoma of the anal canal. Int J Radiat Oncol Biol Phys 1997;37:1101-5. 
30. Pocard M, Tired E, Nugent Delni N, Paec R. Results of salvage abdominoperineal resection for anal cancer after radiotherapy. Dis Colon Rectum 1998;41:148893.

31. Rich TA, Ajani JA, Morrison WH, Ota D, Levin B. Chemoradiation therapy for anal cancer: radiation plus continuous infusion of 5-fluorouracil with or without cisplatin. Radiother Oncol 1993;27:209-15.

32. Ryan DP, Compton CC, Mayer RJ. Carcinoma of the anal canal. N Engl J Med 2000;342:792-800.

33. Shark B, Cunningham JD, Kelsen DP. Cancer of the canal anal region. In: DeVita VT, Hellman S, Rosenberg AS, editors. Cancer. Principles and practice of oncology. 5.ed. Philadelphia: Lippincott-Raven; 1997. p.1234-48.

34. Sischy B. The use of radiation therapy combined with chemotherapy in the management of squamous cell carcinoma of the anus and marginally resectable adenocarcinoma of the rectum. Int J Radiat Oncol Biol Phys 1985;11:1587-93.
35. Sischy B, Doggett RL, Krall JM. Definitive irradiation and chemotherapy for radiosensitization in management of anal carcinoma: interim report on Radiation Therapy Oncology Group study no. 8314. J Natl Cancer Inst 1989;81:850-6.

36. UKCCCR Anal Cancer Trial Working Party. Epidermoid anal cancer: results from the UKCCCR randomised trial of radiotherapy alone versus radiotherapy, 5-fluorouracil, and mitomycin. Lancet 1996;348:1049-54.

37. Watemberg S, Landau O, Avrahmi R, Kaplan I, Giler S, Kott I. Successful treatment of anal tumours with $\mathrm{CO} 2$ laser in elderly, high risk patients. J Clin Laser Med Surg 1996;14:115-7. 